\title{
Molecular Subtyping and Prognostic Prediction Based on the Ferroptosis-Related Long Non-Coding RNA Signature in Clear Cell Renal Cell Carcinoma
}

zhenpeng zhu

Peking University First Hospital https://orcid.org/0000-0003-4283-310X

\section{Cuijian Zhang}

Peking University First Hospital

Jinqin Qian

Peking University First Hospital

Ninghan Feng

Wuxi No 2 People's Hospital

Weijie Zhu

Peking University First Hospital

\section{Yang Wang}

Wuxi No 2 People's Hospital

\section{Yanqing Gong}

Peking University First Hospital

\section{Xuesong Li}

Peking University First Hospital

Jian Lin

Peking University First Hospital

Liqun Zhou ( $\square$ zhoulqmail@sina.com )

Peking University First Hospital

\section{Primary research}

Keywords: Ferroptosis, clear cell Renal Cell Carcinoma, Molecular subtyping, Prognostic signature, Long Noncoding RNA

Posted Date: September 20th, 2021

DOl: https://doi.org/10.21203/rs.3.rs-789595/v1

License: (c) (1) This work is licensed under a Creative Commons Attribution 4.0 International License.

Read Full License 


\section{Abstract}

\section{Background}

Renal cell carcinoma is gradually characteristic of the accumulation of lipid-reactive oxygen species. And, the ferroptosis-related LncRNAs have been reported strongly correlated with tumorigenesis and progression. However, the further functions of ferroptosis-related LncRNAs in clear cell renal cell carcinoma have not been explored.

\section{Methods}

After sample cleaning, data integration, and batch effect removal, we used the Cancer Genome Atlas (TCGA) and International Cancer Genome Consortium (ICGC) public database to screen out the expression and prognostic value of ferroptosis-related LncRNAs and then performed the molecular subtyping. The molecular subtyping was developed using the K-means. Then, the KEGG pathway enrichment and statistical analyses of the immune microenvironment and clusters were carried out. The outcomes indicated significant differences in the immune microenvironment and potential therapeutic targets between the two clusters. Then, LASSO Cox regression was performed to establish the 5LncRNAs-based prognostic signature. The signature could accurately predict patient prognosis and be used as an independent clinical risk factor. And the 5 LncRNAs were validated in the cell lines and clinical samples. We then combined significant clinical parameters in multivariate Cox regression and prognostic signature to construct a clinical predictive nomogram, which provides appropriate guidance for predicting the overall survival of ccRCC patients.

\section{Results}

The prognostic DEFRLncRNAs were identified, and 5 LncRNAs were finally utilized to establish the prognostic signature in the TCGA cohort, which was subsequently validated in the internal and external cohorts. Moreover, we conducted the molecular subtyping and divided the patients in the TCGA cohort into two clusters, showing the difference in the Hallmark pathways, immune infiltration, expression of the immune target, and drug therapy. Furthermore, the nomogram was established better to predict the clinical outcomes of the ccRCC patients.

\section{Conclusions}

Our study conducted molecular subtyping and established a novel predictive signature based on the ferroptosis-related LncRNAs, which could accurately predict prognosis and potential therapeutic targets in ccRCC patients.

\section{Introduction}

Renal cell carcinoma (RCC) is one of the most common malignant tumors of the urinary system(1). As the major pathologic subtype of RCC, clear cell renal cell carcinoma (ccRCC), especially metastatic 
ccRCC, often means high morbidity and mortality(2). To our best knowledge, almost $25 \%-30 \%$ of ccRCC patients are found metastasis at initial diagnosis, which usually means poor prognosis. Moreover, the tumor node metastasis (TNM) staging system, currently applied in clinical practice, is considered less accurate in evaluating the prognosis and progression of ccRCC patients(3). Meanwhile, $73 \%-75 \%$ of identified ccRCC driver aberrations were subclonal, which might contribute to different clinical outcomes $(4,5)$. Hence, performing suitable molecular subtyping and exploring new prognostic signatures to diagnose and evaluate the prognosis of the ccRCC patients remains significant.

During the past decades, ferroptosis has been gradually identified as an iron-dependent, nonapoptotic cell death mode characterized by the accumulation of lipid reactive oxygen species $(6,7)$. Increasingly studies have shown that dysregulation of ferroptosis-related genes (FRGs) plays important role in the occurrence and development of many diseases, especially cancer(8-10). The studies related to ferroptosis have become the focus in treating and detecting related diseases. Meanwhile, as the noncoding RNA plays an emerging role in cancer, long non-coding RNAs (LncRNAs) have been a hot topic of research (11). Interestingly, increasing studies have demonstrated that LncRNAs could participate in the ferroptosis process and then influence tumor development(12-14). Therefore, probing ferroptosisassociated LncRNAs might provide new ideas and sights for ccRCC treatment and prediction.

In this study, we first explored the potential biological functions and associations of ferroptosis-related genes. Afterward, we screened the prognostic ferroptosis-related LncRNAs and conducted the molecular subtyping in the TCGA database. The correlation between the molecular cluster and immune infiltration was explored. Afterward, we conducted the LASSO Cox regression to establish a 5 -ferroptosis-related LncRNAs signature in CCRCC patients and validated it in the ICGC database. Further, the nomogram was conducted integrating the prognostic signature and significant clinical parameters. The results showed that a good predictive performance to the overall survival (OS) of ccRCC patients.

\section{Materials And Methods}

\section{Data selection and processing}

The ccRCC sequencing data (HTseq-FPKM) and the latest corresponding clinical information (Supplementary Table 1) were downloaded from The Cancer Genome Atlas (TCGA) database (https://cancergenome.nih.gov/), including $539 \mathrm{ccRCC}$ samples and 72 normal controls. Meanwhile, we downloaded the transcriptome profile and corresponding survival data from the International Cancer Genome Consortium (ICGC) database (http://daco.icgc.org/) as the validation cohort. Then, we distinguished between IncRNA and mRNA using the human GTF annotation file. The sequencing data processing was conducted via limma and sva packages in $\mathrm{R}(\mathrm{v}$ 4.0.3).

\section{Cell lines and clinical specimens.}

The 293, HK-2, 786-0, 769-P, ACHN, A498, OS-RC-2, and Caki-1 cells were obtained from the American Type Culture Collection (ATCC). All cells were maintained in RPMI-1640 (Corning, USA) or high-glucose 
DMEM (Gibco, USA) with 10\% fetal bovine serum (BI, Israel) and 1\% Penicillin and Streptomycin (Gibco, USA) at $37^{\circ} \mathrm{C}$ and $5 \% \mathrm{CO}_{2} .10$ paired $\mathrm{CCRCC}$ and adjacent normal tissues were obtained from ccRCC patients undergoing surgical resection at Peking University First Hospital. The detailed information of 10 paired tissue specimens were shown in the Supplementary Table 2. The Ethics Committee approved this study of PUFH, and all patients signed informed consent. All procedures were performed according to the World Medical Association Declaration of Helsinki.

\section{Real-time quantitative PCR (qPCR)}

Total RNA of 10 paired clinical samples and six cell lines were extracted by Takara kit according to the manufacturer's protocol. Then, the RNA was reversed to cDNA in a 20ul reaction system. The quantitation of all gene transcripts was done by qPCR using SYBR Premix ExTaq kit, and TUBA was used as a normalized control. The primer sequences were listed in the supplementary Table 3 . Each reaction was performed four times, and the $2^{\wedge-\triangle \triangle C T}$ method was used to calculate the relative mRNA expression level.

\section{Identification of the prognostic ferroptosis-related differentially expressed LncRNAs}

According to the former studies(15-17), we obtained 259 FRGs, and the list was shown (Supplementary Table 4). After that, we screened the ferroptosis-related LncRNAs with the filter (Correlation $>0.5, p$-value $<$ 0.01), including 2854 LncRNAs. The limma package was conducted to determine the differentially expressed LncRNAs (DELncRNAs) between the cCRCC patients and normal controls, including 1333 LncRNAs(18). We intersected the DELncRNAs and ferroptosis-related LncRNAs to obtain the ferroptosisrelated DELncRNAs. Univariate Cox regression was performed in the ferroptosis-related DELncRNAs with overall survival time. Those with $p$-value $<0.01$ were considered prognostic ferroptosis-related DELncRNAs.

\section{Molecular subtyping in ccRCC based on the prognostic ferroptosis-related DELncRNAs}

After obtaining the prognostic ferroptosis-related DELncRNAs (FRDELncRNAs), we performed consensus clustering to identify the molecular subtypes of ccRCC by using the "ConsensusClusterplus" R package(19). We selected $80 \%$ of the prognostic ferroptosis-related DELncRNAs resampling 100 times and determined clusterings of specified cluster counts $(k)$. Following this, the pairwise consensus values were calculated and stored in a symmetrical consensus matrix for each $k$. The $k$, at which there is no appreciable increase, was determined by the cumulative distribution function (CDF) plot and delta area plot. The alteration of Immune infiltration between different clusters was estimated using the Cibersoft method.

\section{Potential biological functions enrichment}

To gain insights into the cellular functions directly regulated by FRGs transcriptional control, we compared the list of FRGs to the biological pathways annotated by the Kyoto Encyclopedia of Genes and Genomes (KEGG)(20). Afterward, according to the two clusters, Gene Set Variation Analysis (GSVA) was conducted using the package of the same name in R software v.4.0.3 to investigate the enrichment 
of HALLMARK pathways with the h.all.v7.4.symbols.gmt gene set from the Molecular Signature Database(21).

\section{Potential therapeutic targets analysis}

Since targeted drugs are commonly used to treat advanced kidney cancer, we used the R package "pRRophetic" to estimate drug response as determined by the half-maximal inhibitory concentration (IC50) for each kidney cancer patient on the Genomics of Drug Sensitivity in Cancer (GDSC) website(22). Further, based on the TCIA database, the Immunophenoscore (IPS) was calculated depending on immune therapy data(23).

\section{Construction of the prognostic predictive risk signature}

Firstly, the TCGA cohort patients were randomly divided into the training set and internal validation set. Meanwhile, the patient in the ICGC cohort was used as the external validation cohort. Based on the prognostic ferroptosis-related DELncRNAs, we constructed the least absolute shrinkage and selection operator (LASSO) Cox regression using the "glmnet" R package. We calculated each patient's riskscore using the regression coefficient score of the individual LncRNAs and their expression value. Besides, we defined the formula for calculating the prognostic risk score as follow: Risk score $=\operatorname{coef}(\operatorname{Lnc} 1)^{\star} \operatorname{Exp}(\operatorname{Lnc} 1)$ $+\operatorname{coef}(\operatorname{Lnc} 2) \star \operatorname{Exp}(\operatorname{Lnc} 2)+\ldots+\operatorname{coef}(\operatorname{Lncn})^{\star} \operatorname{Exp}(\operatorname{Lncn})$. Where "coef" represented the coefficient score estimated by LASSO Cox regression, and "Exp" represented the expression value of the individual LncRNAs. The detailed information from the signature was shown (Table 1). Then, we classified the cCRCC patients into the high- and low-risk groups, according to the median risk score of the training group as the cut-off(24).

\section{Validation of the prognostic risk signature}

We conducted the Kaplan-Meir and receiver operating characteristic (ROC) curve analyses to assess the prognostic risk signature's validity. According to the calculated median risk score, all samples in each group were divided into high- and low-risk groups, and the survminer and timeROC packages were performed to validate the predictive accuracy in the training and validation sets. The area under the curve (AUC) values corresponding to 1-, 3-, and 5-years were calculated. The time-dependent ROC curve was used to validate the predictive performance of the signature. The AUC value of 0.75 or higher was considered the significant predictive value, and the value of 0.60 or higher was regarded as acceptable for prediction. Furthermore, univariate and multivariate Cox regression was conducted to explore if the ferroptosis-signature(FerroSig) could serve as the independent factors.

\section{Construction and validation of the nomogram}

To better predict the prognosis of patients with renal clear cell carcinoma, we established the predictive nomogram based on clinical parameters and prognostic signature(25). Briefly, we first performed univariate and multivariate Cox regression analyses to identify clinical parameters and riskscore that could be used as independent risk factors. Subsequently, the significant factors were used to construct 
the predicted nomogram. We then evaluated the nomogram effect using calibration curves and timedependent ROC curves. The AUC value of 0.75 or higher was considered a significant predictive value, and the value of 0.60 or higher was regarded as acceptable for prediction.

\section{Results}

\section{Identification of the prognostic FRDELncRNAs}

The data processing was performed as described in the methods above. Meanwhile, the flow chart of the whole process was shown (Fig 1). To explore the potential functions of the FRGs, we first conducted the KEGG pathway enrichment analysis. The outcome showed that FRGs were mainly enriched in ferroptosis and autophagy pathways (Fig 2A). Afterward, we explored the FRLncRNAs with the correlation $>0.5$ and p-value $<0.01$, using the limma package. Following this, 1333 DELncRNAs between the CCRCC and normal samples from the TCGA set were screened, and the 5 most obvious up-and down-regulated LncRNAs were labeled (Fig 2B). The overlapped LncRNAs in both DELncRNAs and FRLncRNAs were identified as the FRDELncRNAs. There were 723 LncRNAs for OS selected as prognostic FRDELncRNAs for subsequent analyses (Fig 2C).

\section{Molecular subtyping showed differences in therapeutic choice and immune microenvironments}

The prognostic FRDELncRNAs above were used to screen the molecular subtypes of ccRCC by using ConsensusClusterPlus packages. K-means method was performed for clustering, and $80 \%$ of the LncRNAs were sampled 100 times using the resampling model. The consensus CDF and delta area were calculated to determine the clustering outcomes, as shown in Fig 3B. When cluster number was 2, there was no significant increase in the area under the CDF curve. Hence, we finally divided the samples in the TCGA cohort into cluster 1 and cluster 2. The representative consensus matrix of two clusters (Fig 3A) displayed a well-defined 2-block structure. Principal component analysis (PCA) showed that the samples from two clusters could be well separated (Fig 3C). Afterward, the heatmap integrating the expression of the prognostic FRDELncRNAs and clinical parameters in each subtype were shown (Fig 3D). The results showed that the $\mathrm{pM}$ stage was higher in cluster1 than in cluster, which also clarified that the patients in cluster 1 had a worse prognosis.

\section{Comprehensively analyses of the molecular clusters}

Subsequently, we explored the HALLMARK pathway enrichments alteration between the two clusters using the GSVA method. The results showed that several oncogenic pathways were significantly altered between the two clusters, such as Hypoxia and Apoptosis (Fig 4A). Further, using the pRRophetic packages, we calculated the IC50 between the two clusters for clinical drugs commonly used in ccRCC patients. The outcome indicated that the patients in cluster 1 were more sensitive to Daunorubicin and Tipifarnib. In contrast, using the Dasatinib, Paclitaxel, Sorafenib, and Pazopanib would be more effective for patients in cluster 2 (Fig 4B). 
Since the tumor microenvironment (TME) plays a vital role in the development of tumors, we further explored the correlation between the TME and molecular clusters. As shown in Fig 5A, we first calculated the stromal score, immune score, and ESTIMATE score. Only the immune score showed a significant difference between the two clusters. We then explored the differentially expressed immune cells using the Cibersoft method. Cluster 1 exhibited a higher infiltration of CD8 T cells, regulatory CD4 T cells, and Neutrophils. In comparison, cluster 2 showed a higher infiltration of macrophages (Fig 5B). Meanwhile, the expression level of some potential immunotherapy targets changed significantly in both classifications. Potential immune therapeutic targets such as BRAF and PD-1 expression levels were markedly higher in cluster 1 (Fig 5C). Next, we explored the TCIA database for differences in the presence of immunotherapeutic targets. The results showed Cluster 1 patients were more effective when treated with CTLA4, whereas cluster 2 patients were more effective when receiving the combination of CTLA4 and PD1. (Fig 5D).

\section{Construction and validation of the prognostic signatures based on the prognostic FRDELncRNAs}

Based on the prognostic FRDELncRNAs obtained from the univariate Cox regression analysis, we constructed a 9-FRDELnCRNAs based prognostic signature using the LASSO regression (Fig 6A and $6 \mathrm{~B})$. The detailed information of the LncRNAs from the signature was shown in Table 1 . The risk score is constructed according to the following formula: risk score $=0.058($ LINC00460 $)+0.088($ LINC00894 $)+$ 0.118 (VPS9D1-AS1) + 0.013 (CYTOR) + 0.075 (FOXD2-AS1). Then, based on the calculated median risk score cut-off, patients were divided into the high- and low-risk group. The risk score distribution, survival status, and expression of LncRNAs from the signatures are exhibited in the TCGA training cohort, TCGA validation set, and ICGC validation set (Fig $6 \mathrm{C}$-E). The Kaplan-Meier log-rank test and the time-dependent ROC curve were used to evaluate the predictive ability and accuracy of the prognostic signature. The outcome of the Kaplan-Meier log-rank test showed that the high-risk group had a significantly worse OS compared with the low-risk group in the TCGA training set (Fig 7A), TCGA validation set (Fig 7B), and ICGC validation set (Fig 7C). Meanwhile, the time-dependent ROC curve proved the 1-year, 3-year, and 5year predictive accuracy of the signature for OS (Fig 7A-7C).

\section{Construction and validation of the prognostic nomogram}

After establishing and validating the signatures based on the prognostic FRDELncRNAs, univariate and multivariate Cox regression analyses were used to explore independent risk factors in the TCGA dataset. As shown in Table 2, univariate Cox regression analysis showed that AJCC stage, ISUP grade, Age, and RiskSig were significantly correlated with OS (Table 2). Meanwhile, the multivariate Cox regression analyses of the clinical parameters above, AJCC stage, ISUP grade, Age, and RiskSig, were significantly correlated with OS (Table 2).

To better assess patient prognosis and guide clinical decision-making, we established the nomogram integrating the risk signature and significant clinical parameters in the multivariate Cox regression analyses (Fig 8A). The C-index showed a good agreement of 0.773 , and an established nomogram was shown (Fig 8B). The calibration curves showed that the predictive nomogram could well predict the 
survival status of patients at 1,3 , and 5 years (Fig $8 \mathrm{C}$ ). Also, the nonogram showed better predictive value than clinical indicators and ferroptosis-related signatures (Fig 8D).

\section{Validation of the LncRNAs in cell lines and clinical specimens.}

We finally validated the 5 LncRNAs in signature in ccRCC clinical samples and RCC cell lines. Compared to the normal cell line HK-2, the expression levels of LncRNAs in RCC cell lines were inconsistent, with higher or lower levels present (Fig 9A). The results may be due to the inability of a single cell line to mimic the overall situation of RCC and adjacent normal tissue. We then examined the expression levels of the corresponding mRNAs in 10 paired clinical samples, in which LNC000894, VPS9D-AS1, CYTOR were highly expressed in cancer, while LNC000460 and FOXD2-AS1 were not evident (Fig 9B).

\section{Discussion}

Clear cell Renal Cell Carcinoma is a molecularly heterogeneous tumor characterized as radiotherapy and chemotherapy-resistant $(2,5)$. With the development of diagnostic and therapeutic techniques, the 5-year survival rate of ccRCC patients has been significantly improved. However, $25 \%-30 \%$ of ccRCC patients were found metastases at initial diagnosis(4). The 5-year survival rate is merely $10 \%$. Most ccRCC patients had no apparent symptoms such as pain and hematuria, usually resulting in diagnostic difficulty in the early stage. Moreover, the current TNM staging system used in clinical practice always lacked accuracy for prognostic evaluation. Early diagnosis and accurate assessment of ccRCC patients remains challenging for the reasons mentioned above. Therefore, it is still vital to identify new clinical and molecular biomarkers.

Ferroptosis processes were involved in essential roles in the progression and tumorigenesis of RCC(26). Of note, the expression levels of various iron-related genes were significantly correlated with ccRCC patient prognosis, suggesting that targeting ferroptosis could be an effective option for ccRCC treatment $(27,28)$. Moreover, LncRNAs played important roles in regulating the expression of ferroptosisrelated genes and the process of ferroptosis $(29,30)$. Hence, in this study, we comprehensively analyzed the expression and prognosis of ferroptosis-related LncRNAs. We subsequently molecularly typed and developed a prognostic model based on nine LncRNAs in patients with CCRCC, using the method above.

Although many molecular subtyping of ccRCC based on gene expression has been proposed in recent years, the cluster of LncRNAs associated with ferroptosis has not been fully explored(31). Therefore, we divided ccRCC patients into two clusters based on prognostic DEFRLncRNAs using the NMF algorithm. PCA analysis showed significant differences between the two clusters. The K-M plot showed that cluster1 had a worse prognosis than cluster2. Since the immune components of the tumor microenvironment and immune cells significantly regulate tumor development(32). We further compared the differences in immune cell infiltration between the two clusters. We found that the CD8 + T cell, $T$ cell regulatory, $T$ cells follicular helper, and B cells memory were presented high expression levels in cluster 1 . In contrast, the expression levels of neutrophils and macrophages were significantly increased in cluster 2. Unlike most cancer types, previous studies have shown that the density of CD8 T-cells correlates with poor prognosis 
in patients with $\operatorname{RCC}(33,34)$. This also explains why patients in cluster 1 have a worse prognosis. Moreover, the infiltration of mesenchymal cells and neutrophils may serve as a protective factor for RCC. We then compared the variations in some of the potential immune therapeutic targets. The results the patients in cluster 1 might be more sensitive to the immune therapy. These results imply molecular subtyping for individualized treatment of patients.

Afterward, we established the predictive signature using LASSO regression. K-M plot and time-dependent ROC curves showed that the predictive signature exhibited good predictive performance. Furthermore, most of the LncRNAs in our signature had been reported in various cancer types. LINC00460 has been extensively studied in cancer and shown in several studies to be a prognostic target in renal clear cell carcinoma(35-37). LINC00894 was reported to promote breast cancer metastasis by regulating ZEB1(38). CYTOR and VPS9D1-AS1 were associated with multiple cancer prognoses and could regulate the progression of multiple cancers through sponge miRNAs(39-41). Similarly, FOXD2-AS1 was strongly associated with the prognosis and progression of cancer patients in various cancers $(42,43)$. These validation results in multiple datasets and literature mining results, indicating that the prognostic signature predicts the prognosis of ccRCC patients and may function as the regulator of CCRCC progression.

Indeed, the whole article has several certain limitations. First of all, our data are based on TCGA and GEO databases, and more independent datasets are needed for testing and validation. Secondly, some LncRNAs in our signature play an essential role in cancer and need to be validated in future experiments. In conclusion, we first systematically analyzed the expression and prognostic value of ferroptosis-related LncRNAs and assessed immune infiltration and potential prognostic targets by molecular subtyping of ccRCC patients.

\section{Conclusion}

In conclusion, our study identified the FRDELncRNAs and successfully constructed an individualized cCRCC signature (riskScore), which proved to be significantly correlated with OS in both the training and validation cohorts. We also estimated the potential relationship between immune cell infiltration, immunotherapy-related targets, and potential therapeutic drugs between molecular subtyping clusters. Our research was anticipated to provide new insights into ferroptosis-related LncRNAs for future work.

\section{Abbreviations}

RCC: Renal cell carcinoma

ccRCC: Clear cell renal cell carcinoma

TNM: Tumor node metastasis

LncRNAs: Long non-coding RNAs 
FRGs: Ferroptosis-related genes

TCGA: The Cancer Genome Atlas

GEO: Gene Expression Omnibus

DELncRNAs: Differentially expressed LncRNAs

FRDELncRNAs: Ferroptosis-related differentially expressed LncRNAs

KEGG: Kyoto Encyclopedia of Genes and Genomes

GSVA: Gene Set Variation Analysis

GDSC: Genomics of Drug Sensitivity in Cancer

LASSO: Least absolute shrinkage and selection operator

ROC: Receiver operating characteristic

AUC: area under the curve

OS: Overall survival

\section{Declarations}

Ethics approval and consent to participate

Not Applicable

Consent for publication

Not Applicable

\section{Availability of data and materials}

Public databases analyzed in this study could be found here: TCGA (https://portal.gdc.cancer.gov/) and GEO database (https://www.ncbi.nlm.nih.gov/geo/). The authors will provide the original data supporting the conclusions of this paper without undue reservation.

\section{Competing interests}

The authors declare that there are no conflicts of interest.

\section{Funding}


This study was supported by grants from the National Natural Science Foundation of China [81670617, 81672546, 81602253, 81772703, 81872083], Natural Science Foundation of Beijing [7152146, 7172219], and Wuxi "Taihu Talents Program" Medical and Health High-level Talents Project. All authors would like to thank all contributors to the TCGA and GEO projects. Moreover, all authors thank all researchers at the Institution of Urology, Peking University.

\section{Authors' contributions}

L Zhou, J Lin, X Li, S He, Y Gong, and Z Zhu designed and supervised the study, drafted the manuscript, and interpreted the data. $L$ Zhou, $J$ Lin, and $X$ Li provided the funding. S He and $Y$ Gong provided advice and supervision. Z Zhu, X Ji, W Zhu, C Xu, T Cai, C Huang performed the experiments, obtained, and analyzed the data.

\section{Acknowledgments}

We would like to thank the researchers and study participants at the Peking University Institute of Urology for their contributions.

\section{References}

1. Siegel RL, Miller KD, Fuchs HE, Jemal A. Cancer Statistics, 2021. CA Cancer J Clin. 2021,71(1):7-33.

2. Jonasch $\mathrm{E}$, Walker $\mathrm{CL}$, Rathmell WK. Clear cell renal cell carcinoma ontogeny and mechanisms of lethality. Nat Rev Nephrol. 2021,17(4):245-61.

3. Warren AY, Harrison D. WHO/ISUP classification, grading and pathological staging of renal cell carcinoma: standards and controversies. World J Urol. 2018,36(12):1913-26.

4. Turajlic S, Swanton C. Metastasis as an evolutionary process. Science. 2016,352(6282):169-75.

5. Sanfrancesco JM, Cheng L. Complexity of the genomic landscape of renal cell carcinoma: Implications for targeted therapy and precision immuno-oncology. Crit Rev Oncol Hematol. 2017,119:23-8.

6. Li J, Cao F, Yin H-L, Huang Z-J, Lin Z-T, Mao N, et al. Ferroptosis: past, present and future. Cell Death Dis. 2020,11(2):88.

7. Hirschhorn T, Stockwell BR. The development of the concept of ferroptosis. Free Radic Biol Med. 2019,133:130-43.

8. Mou Y, Wang J, Wu J, He D, Zhang C, Duan C, et al. Ferroptosis, a new form of cell death: opportunities and challenges in cancer. J Hematol Oncol. 2019,12(1):34.

9. Sun $Y$, Chen P, Zhai B, Zhang M, Xiang Y, Fang J, et al. The emerging role of ferroptosis in inflammation. Biomed Pharmacother. 2020,127:110108.

10. Liang C, Zhang X, Yang M, Dong X. Recent Progress in Ferroptosis Inducers for Cancer Therapy. Adv Mater. 2019,31(51):e1904197. 
11. Ransohoff JD, Wei Y, Khavari PA. The functions and unique features of long intergenic non-coding RNA. Nat Rev Mol Cell Biol. 2018,19(3):143-57.

12. Mao $C$, Wang $X$, Liu $Y$, Wang $M$, Yan $B$, Jiang $Y$, et al. A G3BP1-Interacting IncRNA Promotes Ferroptosis and Apoptosis in Cancer via Nuclear Sequestration of p53. Cancer Res. 2018,78(13):3484-96.

13. Wang M, Mao C, Ouyang L, Liu Y, Lai W, Liu N, et al. Long noncoding RNA LINC00336 inhibits ferroptosis in lung cancer by functioning as a competing endogenous RNA. Cell Death Differ. 2019,26(11):2329-43.

14. Wang Z, Chen X, Liu N, Shi Y, Liu Y, Ouyang L, et al. A Nuclear Long Non-Coding RNA LINC00618 Accelerates Ferroptosis in a Manner Dependent upon Apoptosis. Mol Ther. 2021,29(1):263-74.

15. Zhu L, Yang F, Wang L, Dong L, Huang Z, Wang G, et al. Identification the ferroptosis-related gene signature in patients with esophageal adenocarcinoma. Cancer Cell Int. 2021,21(1):124.

16. Hassannia B, Vandenabeele P, Vanden Berghe T. Targeting Ferroptosis to Iron Out Cancer. Cancer Cell. 2019,35(6):830-49.

17. Doll S, Freitas FP, Shah R, Aldrovandi M, da Silva MC, Ingold I, et al. FSP1 is a glutathioneindependent ferroptosis suppressor. Nature. 2019,575(7784):693-8.

18. Greenberg SA, Sanoudou D, Haslett JN, Kohane IS, Kunkel LM, Beggs AH, et al. Molecular profiles of inflammatory myopathies. Neurology. 2002,59(8):1170-82.

19. Wilkerson MD, Hayes DN. ConsensusClusterPlus: a class discovery tool with confidence assessments and item tracking. Bioinformatics. 2010,26(12):1572-3.

20. Kanehisa M, Goto S. KEGG: kyoto encyclopedia of genes and genomes. Nucleic Acids Res. 2000,28(1):27-30.

21. Hänzelmann S, Castelo R, Guinney J. GSVA: gene set variation analysis for microarray and RNA-seq data. BMC Bioinformatics. 2013,14:7.

22. Geeleher P, Cox NJ, Huang RS. Clinical drug response can be predicted using baseline gene expression levels and in vitro drug sensitivity in cell lines. Genome Biol. 2014,15(3):R47.

23. Charoentong P, Finotello F, Angelova M, Mayer C, Efremova M, Rieder D, et al. Pan-cancer Immunogenomic Analyses Reveal Genotype-Immunophenotype Relationships and Predictors of Response to Checkpoint Blockade. Cell Rep. 2017,18(1):248-62.

24. Hong W, Liang L, Gu Y, Qi Z, Qiu H, Yang X, et al. Immune-Related IncRNA to Construct Novel Signature and Predict the Immune Landscape of Human Hepatocellular Carcinoma. Mol Ther Nucleic Acids. 2020,22:937-47.

25. Iasonos A, Schrag D, Raj GV, Panageas KS. How to build and interpret a nomogram for cancer prognosis. J Clin Oncol. 2008,26(8):1364-70.

26. Yu H, Guo P, Xie X, Wang Y, Chen G. Ferroptosis, a new form of cell death, and its relationships with tumourous diseases. J Cell Mol Med. 2017,21(4):648-57. 
27. Mou Y, Wu J, Zhang Y, Abdihamid O, Duan C, Li B. Low expression of ferritinophagy-related NCOA4 gene in relation to unfavorable outcome and defective immune cells infiltration in clear cell renal carcinoma. BMC Cancer. 2021,21(1):18.

28. Wang J, Yin X, He W, Xue W, Zhang J, Huang Y. SUV39H1 deficiency suppresses clear cell renal cell carcinoma growth by inducing ferroptosis. Acta Pharm Sin B. 2021,11(2):406-19.

29. Lu J, Xu F, Lu H. LncRNA PVT1 regulates ferroptosis through miR-214-mediated TFR1 and p53. Life Sci. 2020,260:118305.

30. Song X, Long D. Nrf2 and Ferroptosis: A New Research Direction for Neurodegenerative Diseases. Front Neurosci. 2020,14:267.

31. Zhong W, Zhang F, Huang C, Lin Y, Huang J. Identification of Epithelial-Mesenchymal TransitionRelated IncRNA With Prognosis and Molecular Subtypes in Clear Cell Renal Cell Carcinoma. Front Oncol. 2020,10:591254.

32. Xiong $\mathrm{Y}$, Wang Z, Zhou Q, Zeng H, Zhang H, Liu Z, et al. Identification and validation of dichotomous immune subtypes based on intratumoral immune cells infiltration in clear cell renal cell carcinoma patients. J Immunother Cancer. 2020,8(1).

33. Nakano O, Sato M, Naito Y, Suzuki K, Orikasa S, Aizawa M, et al. Proliferative activity of intratumoral CD8(+) T-lymphocytes as a prognostic factor in human renal cell carcinoma: clinicopathologic demonstration of antitumor immunity. Cancer Res. 2001,61(13):5132-6.

34. Giraldo NA, Becht E, Pagès F, Skliris G, Verkarre V, Vano Y, et al. Orchestration and Prognostic Significance of Immune Checkpoints in the Microenvironment of Primary and Metastatic Renal Cell Cancer. Clin Cancer Res. 2015,21(13):3031-40.

35. Yang H, Xiong X, Li H. Development and Interpretation of a Genomic Instability Derived IncRNAs Based Risk Signature as a Predictor of Prognosis for Clear Cell Renal Cell Carcinoma Patients. Front Oncol. 2021,11:678253.

36. Wang Y, Yan K, Wang L, Bi J. Genome instability-related long non-coding RNA in clear renal cell carcinoma determined using computational biology. BMC Cancer. 2021,21(1):727.

37. Zhang S, Zhang F, Niu Y, Yu S. Aberration of IncRNA LINC00460 is a Promising Prognosis Factor and Associated with Progression of Clear Cell Renal Cell Carcinoma. Cancer Manag Res. 2021,13:648997.

38. Meng D-F, Shao H, Feng C-B. LINC00894 Enhances the Progression of Breast Cancer by Sponging miR-429 to Regulate ZEB1 Expression. Onco Targets Ther. 2021,14:3395-407.

39. Zhu H, Shan Y, Ge K, Lu J, Kong W, Jia C. LncRNA CYTOR promotes pancreatic cancer cell proliferation and migration by sponging miR-205-5p. Pancreatology. 2020,20(6):1139-48.

40. Hu B, Yang X-B, Yang X, Sang X-T. LncRNA CYTOR affects the proliferation, cell cycle and apoptosis of hepatocellular carcinoma cells by regulating the miR-125b-5p/KIAA1522 axis. Aging (Albany NY). 2020,13(2):2626-39.

41. Liu H, Zhang X, Jin X, Yang Y, Liang G, Ma Y, et al. Long Noncoding RNA VPS9D1-AS1 Sequesters microRNA-525-5p to Promote the Oncogenicity of Colorectal Cancer Cells by Upregulating HMGA1. 
Cancer Manag Res. 2020,12:9915-28.

42. Liang X, Chen Z, Wu G. FOXD2-AS1 Predicts Dismal Prognosis for Oral Squamous Cell Carcinoma and Regulates Cell Proliferation. Cell Transplant. 2020,29:963689720964411.

43. Xue W, Shen Z, Li L, Zheng Y, Yan D, Kan Q, et al. Long non-coding RNAs MACC1-AS1 and FOXD2AS1 mediate NSD2-induced cisplatin resistance in esophageal squamous cell carcinoma. Mol Ther Nucleic Acids. 2021,23:592-602.

\section{Tables}

Table 1 Detailed information of the LncRNAs in the prognostic signature

\begin{tabular}{lllll} 
Gene & Ensembl ID & Description & Located & Coef \\
\hline LINC00460 & ENSG00000233532 & $\begin{array}{l}\text { Long Intergenic Non-Protein Coding } \\
\text { RNA 460 }\end{array}$ & $13 q 33.2$ & 0.05808 \\
\hline LINC00894 & ENSG00000235703 & EOLA2 Divergent Transcript & Xq28 & 0.08831 \\
\hline $\begin{array}{l}\text { VPS9D1- } \\
\text { AS1 }\end{array}$ & ENSG00000261373 & VPS9D1 Antisense RNA 1 & $16 q 24.3$ & 0.11775 \\
\hline CYTOR & ENSG00000222041 & Cytoskeleton Regulator RNA & $3 q 13.2$ & 0.01316 \\
\hline FOXD2-AS1 & ENSG00000237424 & $\begin{array}{l}\text { FOXD2 Adjacent Opposite Strand RNA } \\
1\end{array}$ & $1 \mathrm{p33}$ & 0.07545
\end{tabular}

Table 2 Univariate and multivariate Cox analyses of clinical parameters and risk signature

Univariate analysis

\begin{tabular}{lllll}
\hline Parameters & HR $(95 \% \mathrm{Cl})$ & $\rho$ value & HR $(95 \% \mathrm{Cl})$ & $\rho$ value \\
\hline Gender & $0.963 \rrbracket 0.703 \rrbracket 1.319 \rrbracket$ & 0.815 & $0.964 \rrbracket 0.700 \rrbracket 1.326 \rrbracket$ & 0.820 \\
\hline AJCC stage & $1.870 \rrbracket 1.638 \rrbracket 2.136 \rrbracket$ & $<0.001$ & $1.597 \rrbracket 1.373 \rrbracket 1.859 \rrbracket$ & $<0.001$ \\
\hline ISUP grade & $2.251 \rrbracket 1.835 \rrbracket 2.763 \rrbracket$ & $<0.001$ & $1.326 \rrbracket 1.051 \rrbracket 1.673 \rrbracket$ & 0.017 \\
\hline Age & $1.690 \rrbracket 1.241 \rrbracket 2.303 \rrbracket$ & $<0.001$ & $1.567 \rrbracket 1.145 \rrbracket 2.145 \rrbracket$ & 0.005 \\
\hline RiskSig & $6.535 \rrbracket 4.608 \rrbracket 9.267 \rrbracket$ & $<0.001$ & $3.733 \rrbracket 2.513 \rrbracket 5.546 \rrbracket$ & $<0.001$
\end{tabular}

\section{Figures}




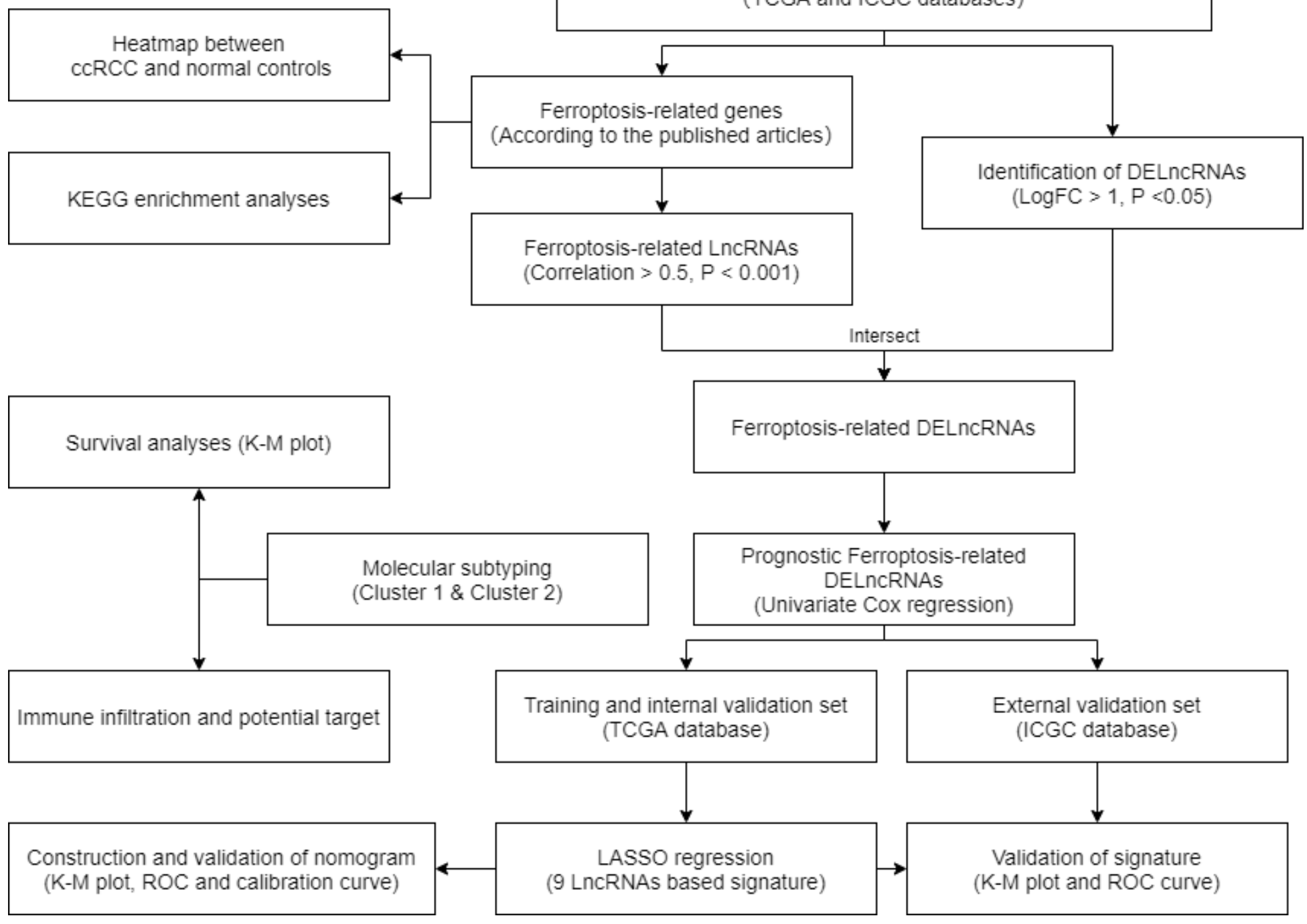

\section{Figure 1}

Flow chart of the whole analysis processes of this study. 

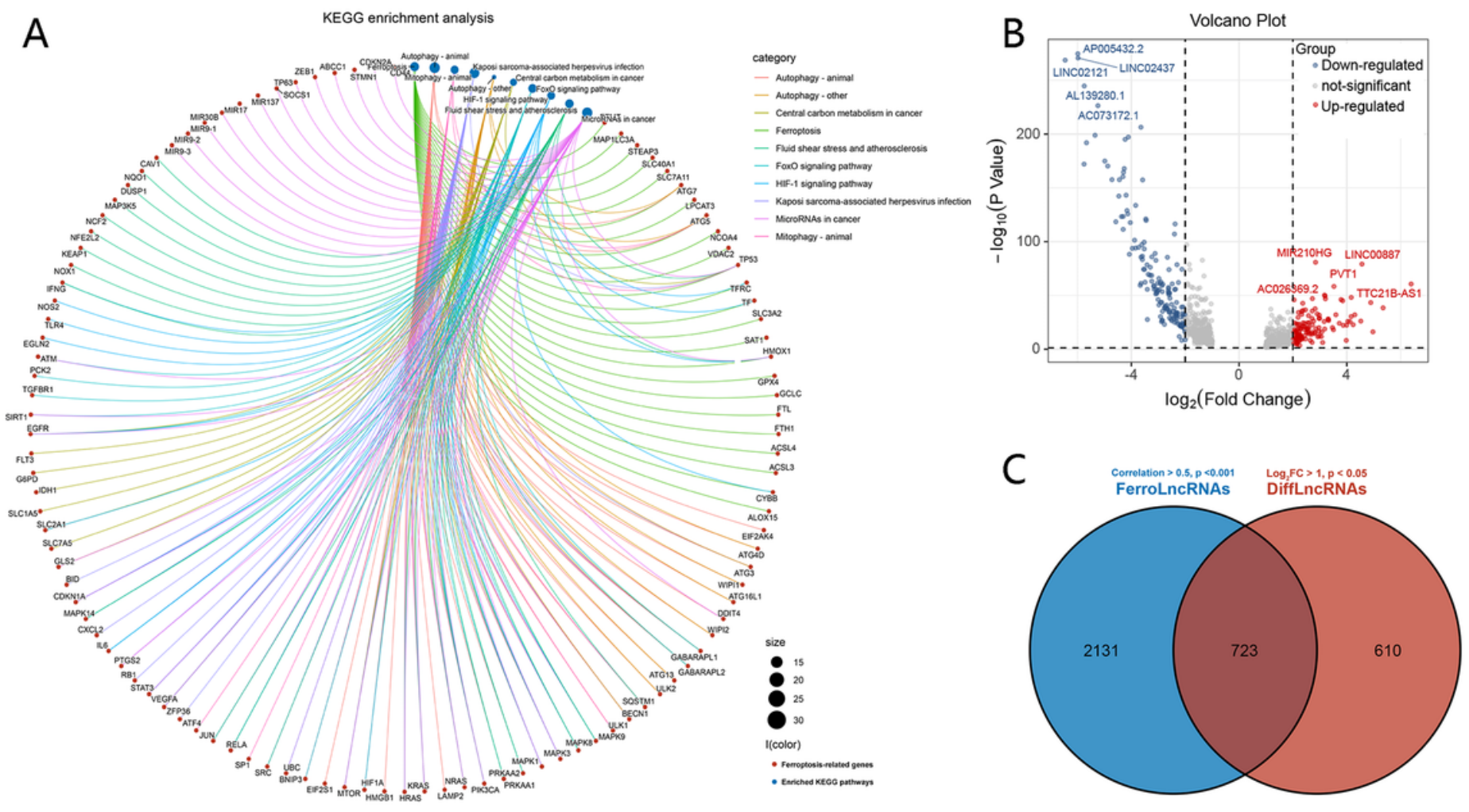

Figure 2

Function annotations of ferroptosis-related genes and Identification of the ferroptosis-related LncRNAs. (A) KEGG enrichment analysis of the ferroptosis-related genes, the larger the shape of the dot, the more corresponding genes are represented. (B) Volcano plot of the differentially expressed ferroptosis-related LncRNAs between the ccRCC and normal samples. The 5 most significant up-and down-regulated LncRNAs were labeled separately. (C) The Venn plot of the overlapped LncRNAs between two cohorts.

A

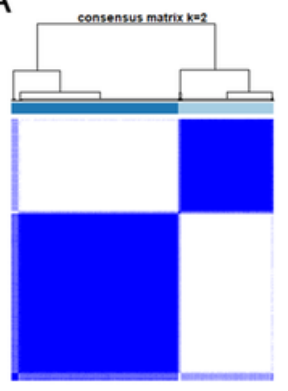

C

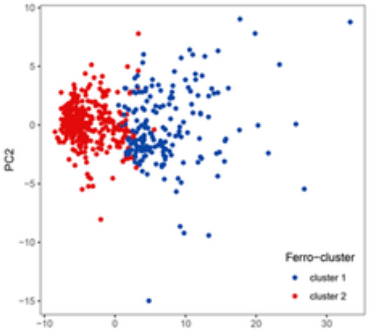

B

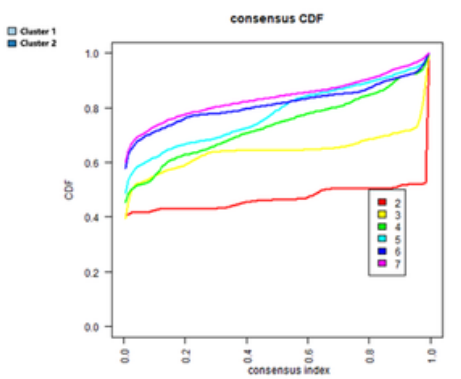

D

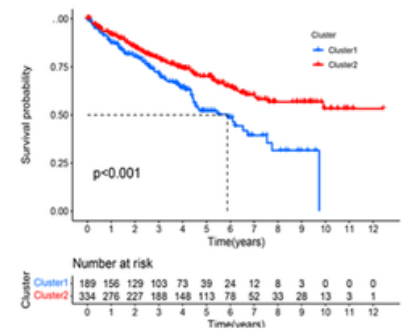

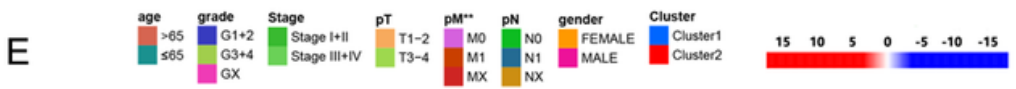

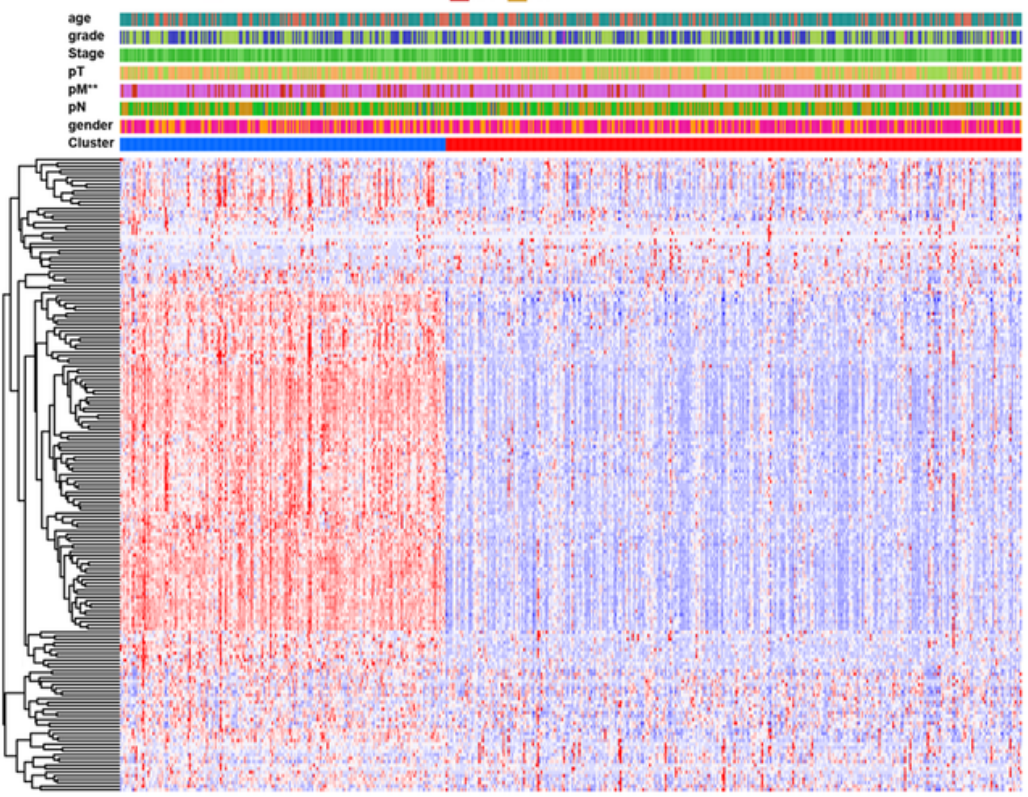


Molecular subtyping of the cCRCC patients based on the prognostic ferroptosis-related LncRNAs. (A) The heatmap corresponding to the consensus matrix for $\mathrm{k}=2$ was obtained by applying consensus clustering. Color gradients represent consensus values from $0-1$, white corresponds to 0 and dark blue to 1 . (B) Consensus among clusters for each category number k. (C) Principal Component Analysis and (D) K-M survival analysis of the two clusters. (E) The composite heatmap corresponding to the cluster and mRNA expression, TNM stage, AJCC stage, and ISUP grade, and Age as the annotations. ${ }^{* *} \mathrm{P}<0.01$.
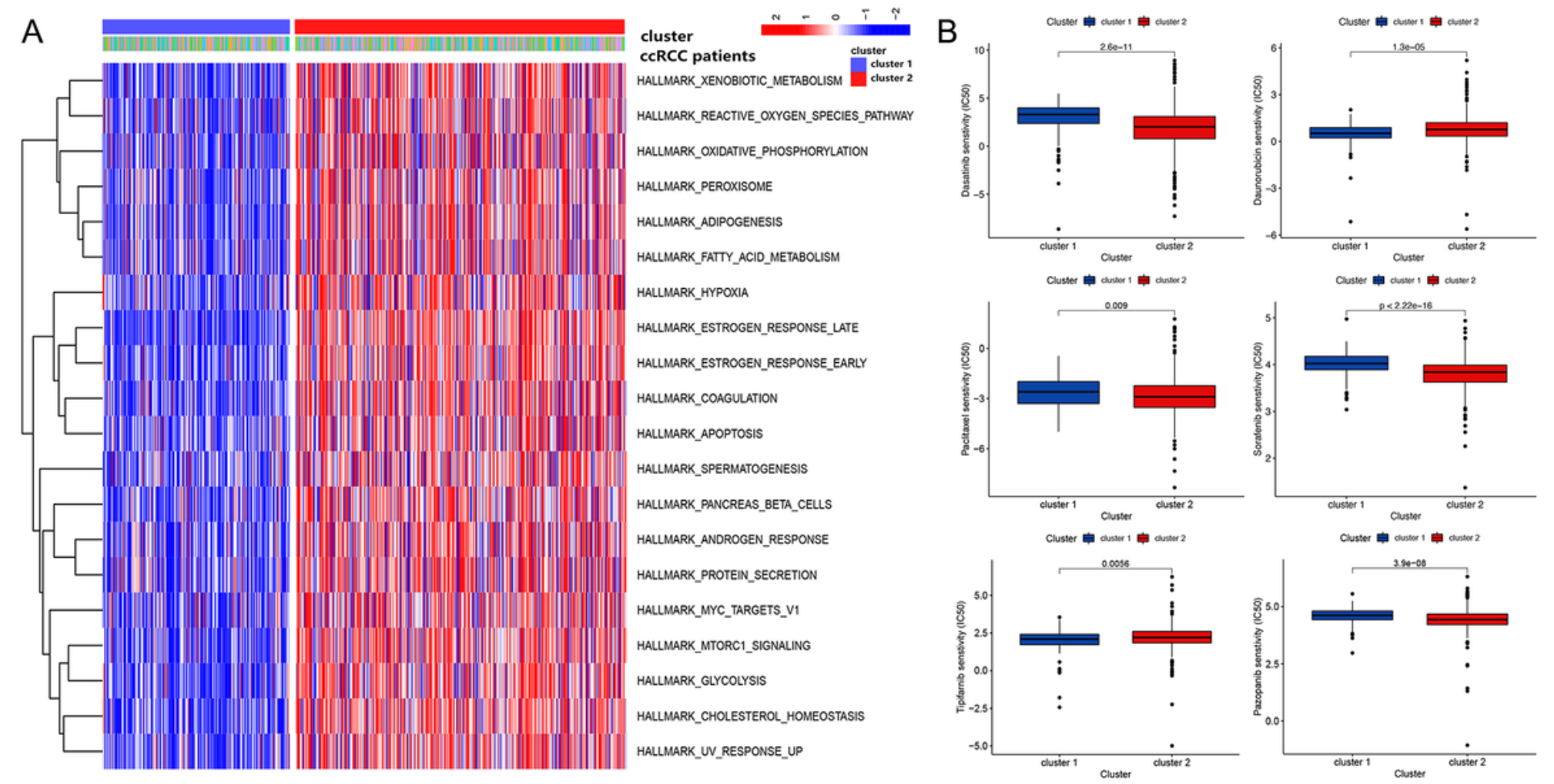

Figure 4

Biological functions and immune infiltration between two clusters. (A) Gene set variation analysis (GSVA) was performed to compute HALLMARK pathways between two clusters. (B) The IC50 data on drugs for ccRCC differentially expressed in the two clusters obtained by applying pRRophetic were shown. 

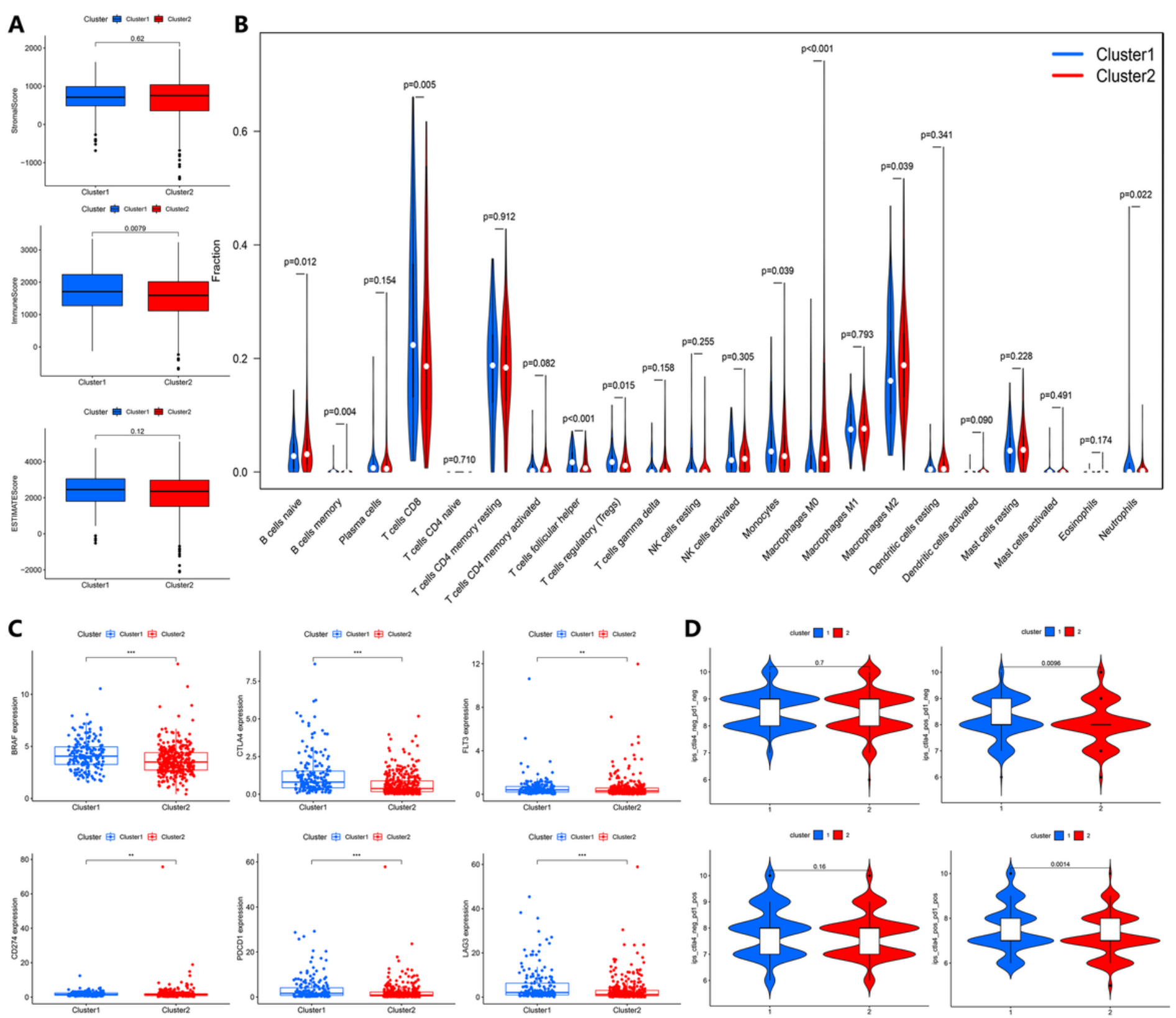

\section{Figure 5}

Tumor environment especially immune infiltration and potential immune therapeutic target between two clusters. (A) Tumor environment scores between two clusters. (B) The differentially expressed immune infiltrated cells in the two clusters obtained by applying Cibersoft were shown. (C) Common potential immune therapeutic targets between two clusters were shown. ${ }^{*} P<0.05,{ }^{\star *} P<0.01,{ }^{\star} * \star P<0.001$ (D) The different expressed of four immune status targets between two clusters were explored from the TCIA database. 

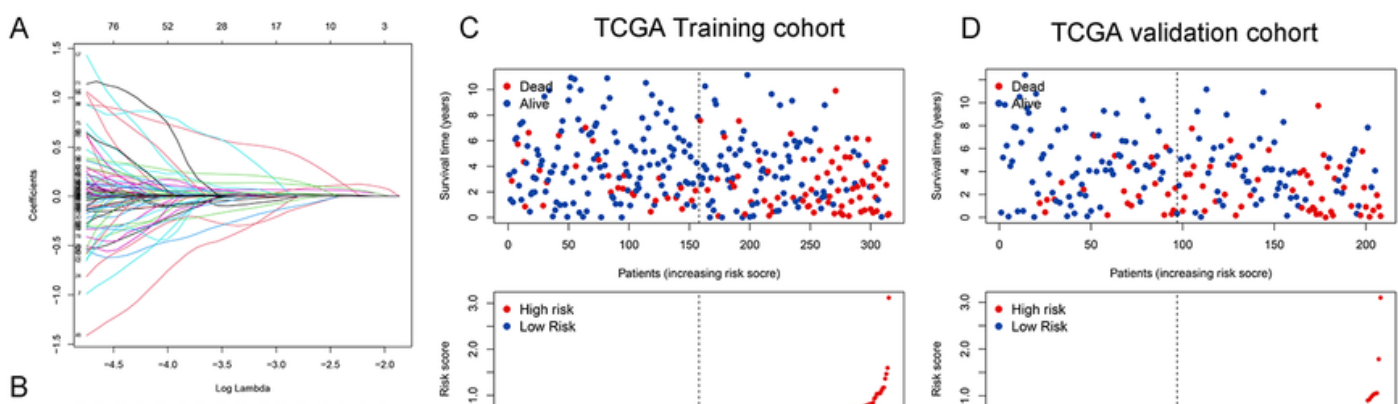

E ICGC validation cohort
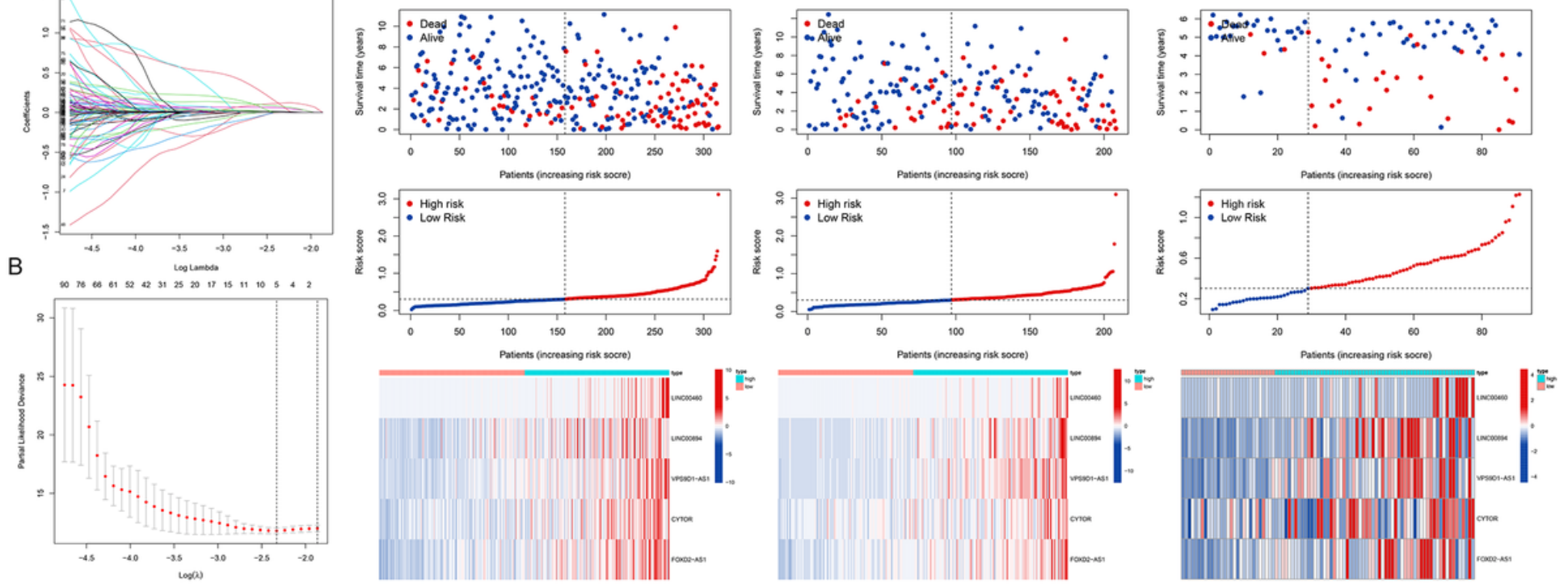

\section{Figure 6}

Establishment of the 9-LncRNAs based prognostic signature. (A) LASSO coefficient profiles of the prognostic DEFRGs. (B) Partial likelihood deviance was plotted versus log (Lambda). The vertical dotted line indicates the lambda value with the minimum error and the largest lambda value. (C-E) LncRNA expression patterns and the distribution of survival status increased risk score in the TCGA training set, TCGA internal validation set, and ICGC external validation set.

A

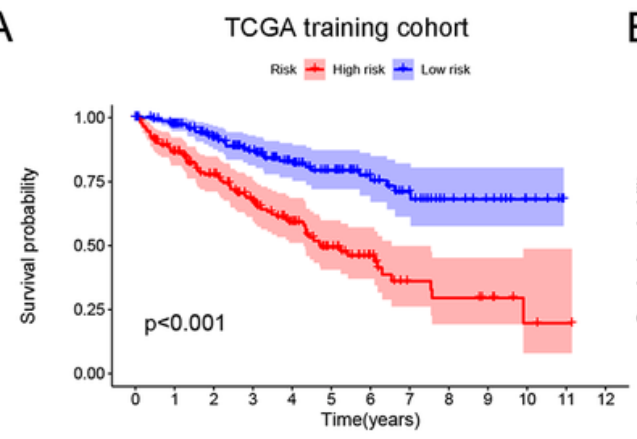

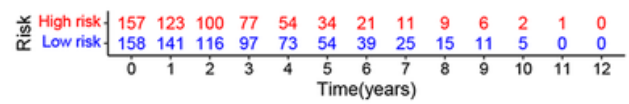

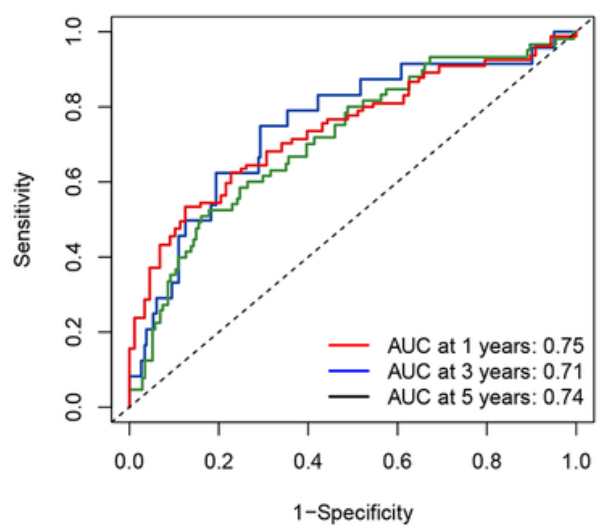

$\mathrm{B}$

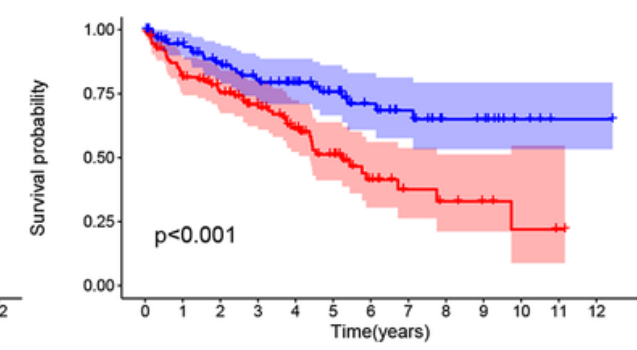

\begin{tabular}{l|lllllllllllll} 
- High risk & 111 & 87 & 71 & 58 & 43 & 27 & 15 & 8 & 6 & 4 & 2 & 1 & 0
\end{tabular}

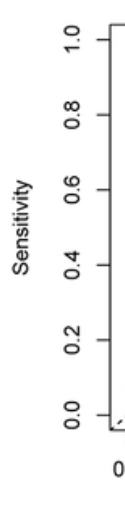

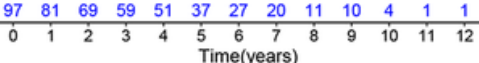

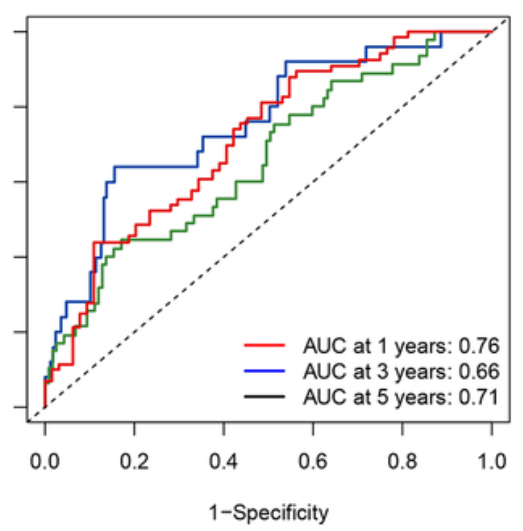

C ICGC validation cohort

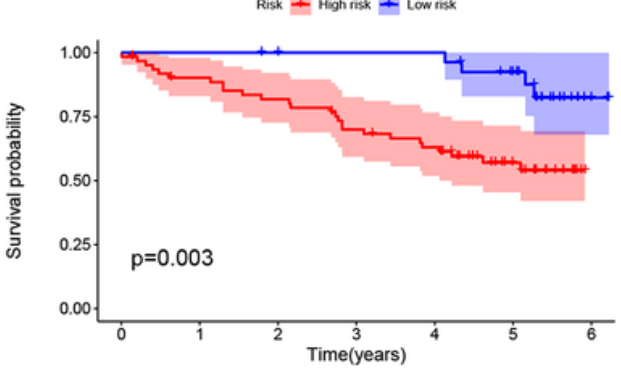

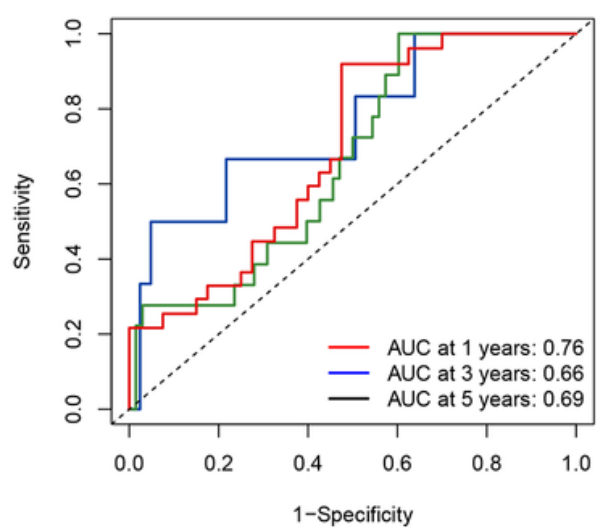

Figure 7 
Validation of the prognostic signature. K-M plot analyses between the high-and low-risk patients in the TCGA training cohort (A), TCGA validation cohort (B), and ICGC validation cohort (C). The 1-, 3-, 5-year time-dependent ROC curves in the TCGA training cohort (A), TCGA validation cohort (B), and ICGC validation cohort (C).

A

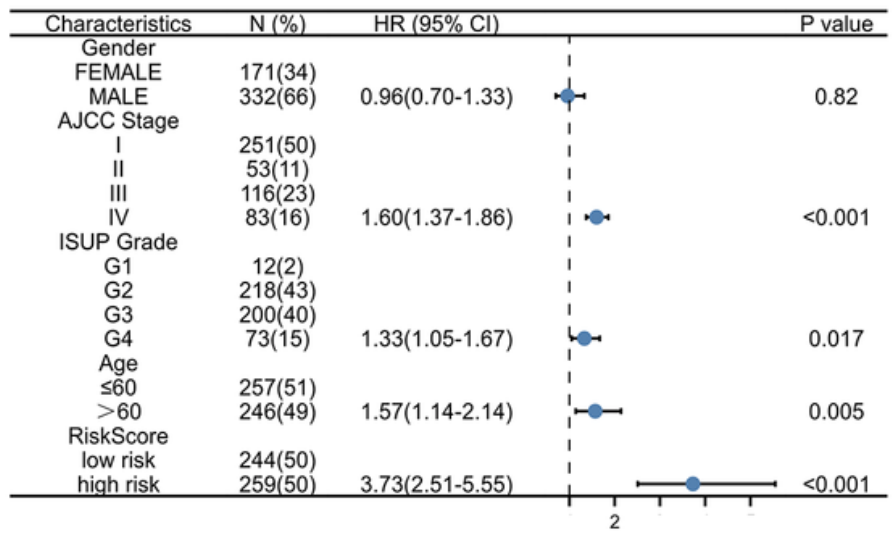

C

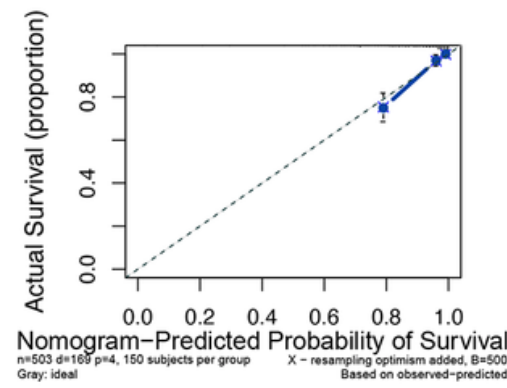

D

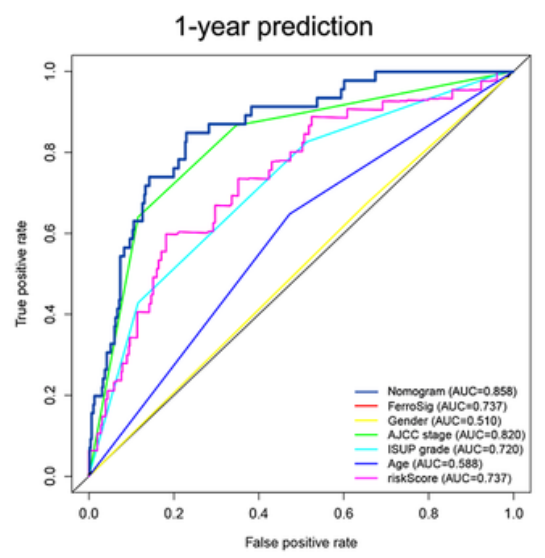

B

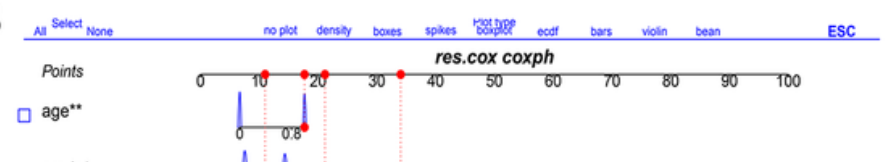

$\square$ grade*

$\square$ stage $* . *$

$\square$ riskScore $\cdots$

Total points

口

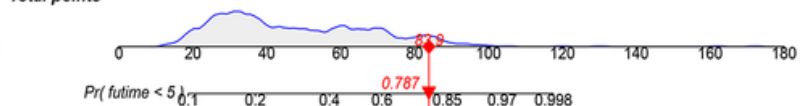

$\begin{array}{llllllll}\text { Prf futime }<3.04 & 0.1 & 0.2 & 0.578 & 0.6 & 0.85 & 0.97 & 0.998\end{array}$

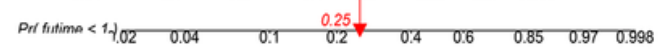
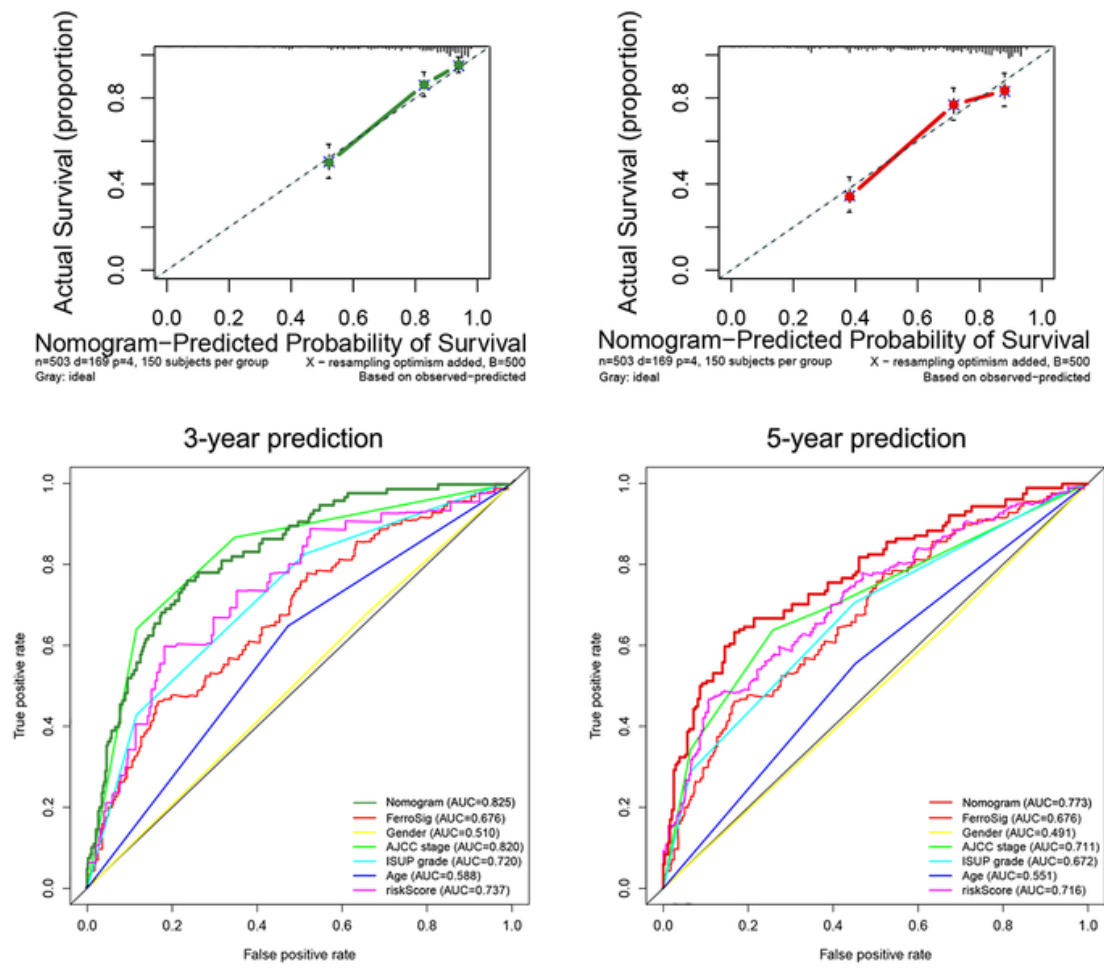

Figure 8

Construction and validation of the prognostic nomogram. (A) Forest plot of the significant clinical parameters in the multivariate Cox regression. (B) The nomogram based on the significant clinical parameters and risk signature (C) Calibration curves of the nomogram for 1-, 3-, and 5-year survival prediction. (D) The predictive value of the nomogram, risk signature, and clinical parameters. 

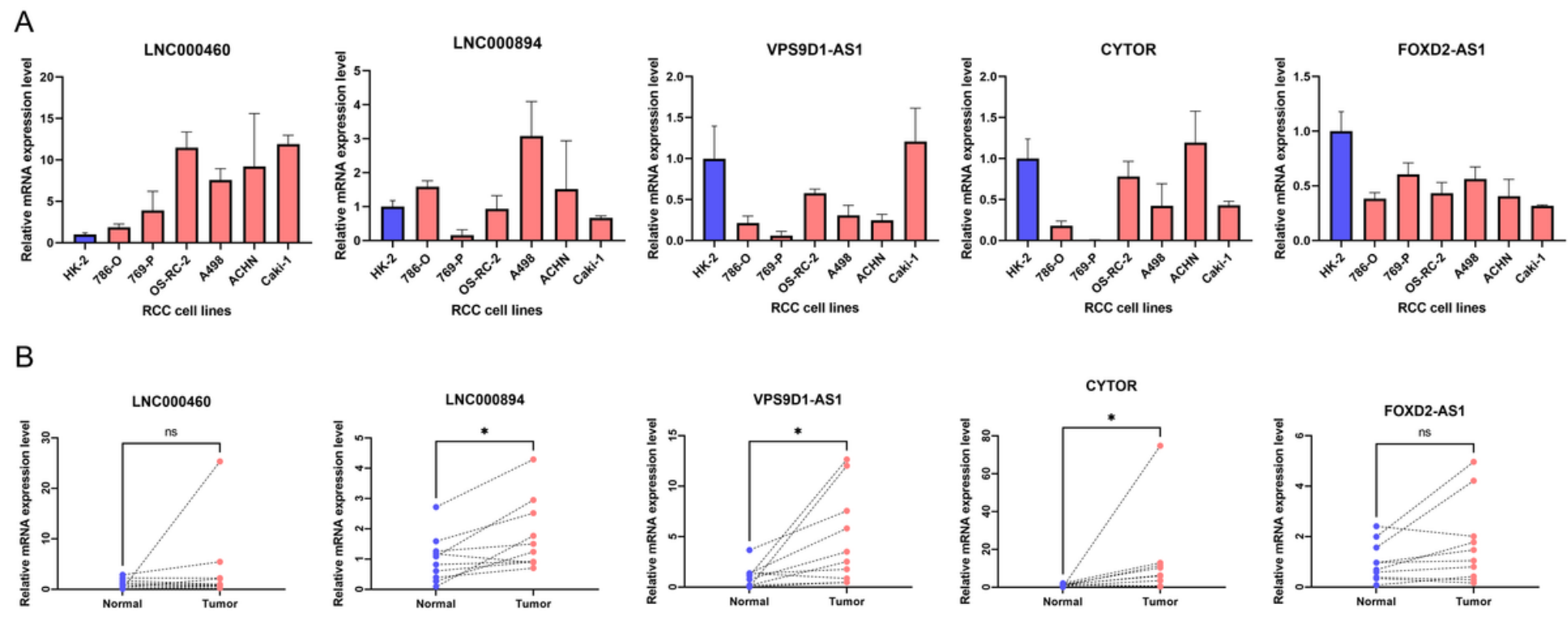

Figure 9

Validation of expression levels of LncRNAs in signaturein cell lines and samples. (A) Levels of mRNA expression of LncRNAs in cell lines. (B) The mRNA expression levels of LncRNAs in 10 pairs of paired clinical samples. ${ }^{*} \mathrm{P}<0.05$.

\section{Supplementary Files}

This is a list of supplementary files associated with this preprint. Click to download.

- SupplementaryFigure1.tif

- SupplementaryTable1.docx

- SupplmentaryTable2.xIsx

- SupplmentaryTable3.xlsx

- SupplmentaryTable4.xlsx 